\title{
Emergence of cancer stem cells in hepatocellular carcinoma
}

\author{
Sahra Pilz \& Gunnar Schotta
}

\begin{abstract}
Liver cancer represents the second most deadly human malignancy. The major histological subtype called hepatocellular carcinoma (HCC) arises by chronic inflammation-triggered regenerative responses of normally quiescent hepatocytes and progenitors, respectively. Such regenerative stress accelerates the accumulation of genetic and epigenetic changes (Yamashita \& Wang, 2013), while detailed mechanisms remain uncertain. In this issue of The EMBO Journal, Nikolaou et al present a novel HCC model that facilitates both isolation and molecular characterization of self-renewing, HCC-propagating cancer stem cells that could instruct future interventions (Nikolaou et al, 2014).
\end{abstract}

See also: KC Nikolaou et al (February 2015)

$\mathrm{T}$ umor cells show heterogeneity in terms of their expression profiles and their epigenetic landscapes. In the recent years, the notion has emerged that some tumor cells possess stemness properties, meaning that they have the ability to self-renew and to generate a whole tumor after transplantation. These so-called cancer stem cells (CSCs) are especially important targets of cancer therapy, since they are thought to orchestrate tumor growth. CSCs are highly resistant against therapeutic measures and have high metastatic and invasive capacity. Therefore, continuous efforts are undertaken to isolate CSCs and to trace their developmental origin(s) (Yamashita \& Wang, 2013; Kreso \& Dick, 2014). It is currently assumed that HCC is induced and/or maintained by CSCs, either arising from hepatocyte de-differentiation or HPC transformation. Systematic studies to address these hypotheses are currently lacking (Yamashita \& Wang, 2013; Oishi et al, 2014).

Cancer stem cells emergence from dedifferentiating hepatocytes is supported by experiments that validated their reprogramming capacity. For instance, inactivation of the Hippo pathway, a regulator of cell proliferation, is sufficient to de-differentiate adult hepatocytes into cells with progenitor characteristics (Yimlamai et al, 2014). Furthermore, the activation of the NOTCH pathway in hepatocytes results in their transdifferentiation to biliary epithelial cells (Yanger et al, 2013). Finally, the simultaneous activation of the NOTCH and AKT pathways leads to the onset of intrahepatic cholangiocarcinoma originating exclusively from hepatocytes (Fan et al, 2012).

Support for the HPC-origin of CSCs arises from their phenotypic similarities as well as their common ability to self-renew and to differentiate. Moreover, CSCs found in HCC have been shown to express similar oncofetal marker as HPCs (Yamashita \& Wang, 2013). Those similarities strongly suggest that HPCs could develop into CSCs, while definitive experimental proof was so far missing.

The experiments conducted by Nikolaou et al established a new model for HCC addressing this gap and facilitating direct isolation of CSCs. They functionally analyzed the histone methyltransferase PRSET7 in liver development. PR-SET7 is the sole enzyme that catalyzes histone H4K20 monomethylation (H4K20me1). This methylation plays an important role in many cellular processes like DNA replication, DNA damage response and mitotic condensation (Jørgensen et al, 2013). PR-SET7 knock-out (ko) is lethal in mice and flies and leads to cell cycle arrest and apoptosis (Driskell et al,
2012; Jørgensen et al, 2013). Using a cre-lox system, Nikolaou et al knocked out PR-SET7 in embryonic mouse livers. Consistent with the essential role of PR-SET7, prenatal lethality was observed. E18.5 embryos were anemic and had a severely reduced liver volume. In contrast, mice with PR-SET7 deletion in adult liver survived and only displayed areas of necrotic cell death. Necrosis was found in regenerating areas of the liver and was caused by the attempted proliferation of hepatocytes which die due to the lack of PR-SET7. To stimulate liver regeneration, the authors decided to perform partial hepatectomy (PHx), which leads to compensatory growth of the remaining tissue by proliferation of adult hepatocytes (Fig 1). PR-SET7-deficient cells die upon proliferation, and enhanced necrotic areas in PR-SET7-deficient PHx livers coincided with chronic inflammation. Interestingly, all PRSET7 ko mice with PHx developed HCC between postnatal days 240-300. Thus, PHx in PR-SET7 ko livers reflects the inflammatory aspect of human HCC, although in humans hepatocyte proliferation is not as severely impaired.

Remarkably, the tumor cells isolated by Nikolaou et al showed CSC properties: they could be cultured in vitro over several passages and they could give rise to tumors with characteristics of the parental tumor when engrafted into immunodeficient mice. These properties distinguish them from a previously described HCC model which was only transplantable into already damaged livers (He et al, 2013). Why the tumor progenitor cells isolated by He et al and the CSCs isolated by Nikolaou et al differ, is currently unclear. One possibility is that they might stem from different origins. The inability of PR-SET7-deficient hepatocytes to 


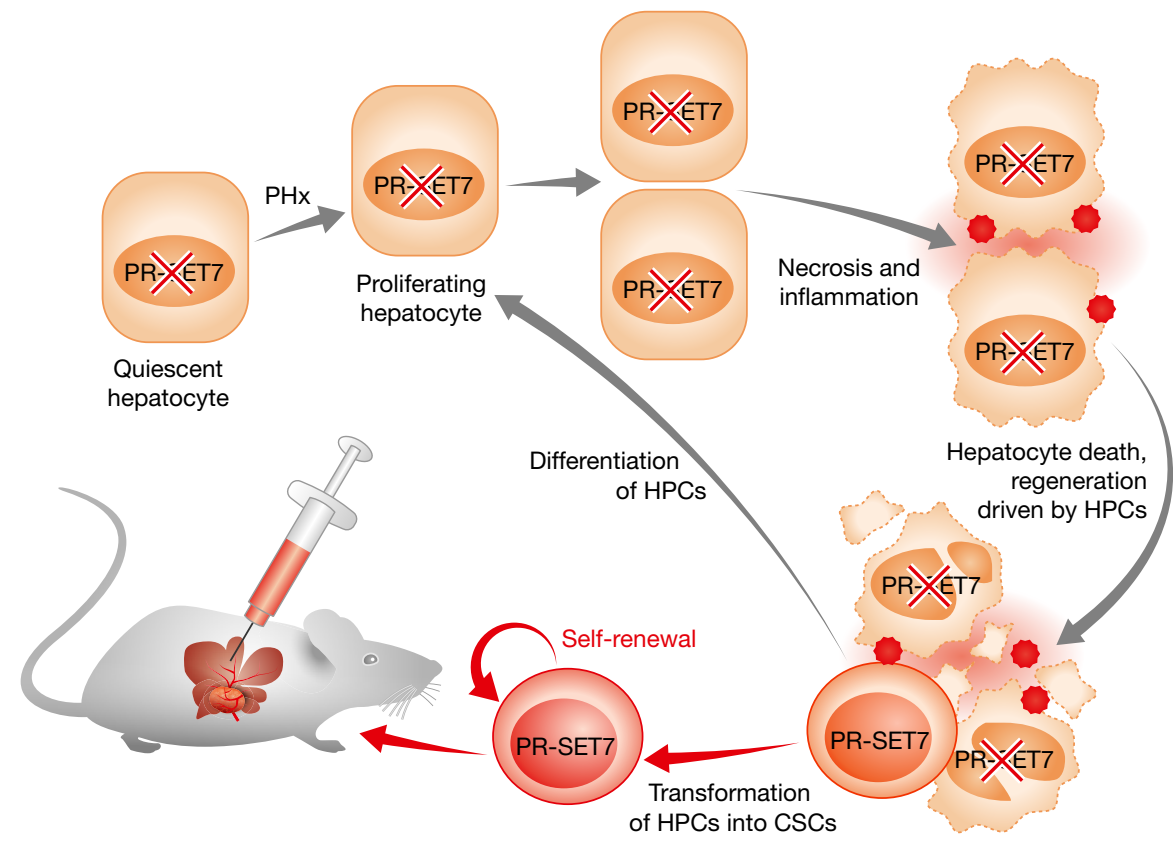

Figure 1. PHX of adult PR-SET7 ko livers leads to HCC onset.

PHX in PR-SET7-deficient livers causes proliferation of normally quiescent hepatocytes. Proliferation of PR-SET7deficient hepatocytes leads to necrotic cell death which is proceeded by an inflammatory response. Thus, liver regeneration involves differentiation of initially PR-SET7-proficient HPCS to hepatocytes. Excessive proliferation of HPCs in combination with chronic inflammation results in the development of HCC. A remarkable feature of these tumors is the presence of CSCS with stemness properties: they can self-renew and they can be transplanted into immunodeficient mice, where they give rise to new tumors.

proliferate and the expression of ductal progenitor markers in CSCs suggest that they originate from HPCs. In contrast, specific induction of DNA damage in hepatocytes and anatomical properties suggest that the tumor progenitor cells isolated by He et al might rather originate from adult hepatocytes. Additional experiments like lineage tracing are required to clarify the origin of these cells.

In summary, Nikolaou et al created a new and very informative HCC model. Arising HCCs present with very high penetrance and surprisingly little tumor heterogeneity. Notably, the tumor cell population includes CSCs that have the ability to self-renew and to initiate tumors when transplanted into immunodeficient mice. Although these cells have been phenotypically and functionally well characterized, we still know very little about their genetic and epigenetic aberrations. Further analyses should reveal CSC-specific oncogenes and tumor suppressor genes. Furthermore, the high penetrance of CSCs in this tumor model will allow for a better understanding of their biological features such as the regulation of proliferation and drivers for their metastatic capacity. Even more intriguing will be potential translation of these animal studies for human HCCs.

\section{References}

Driskell I, Oda H, Blanco S, Nascimento E, Humphreys P, Frye M (2012) The histone methyltransferase Setd8 acts in concert with c$M y c$ and is required to maintain skin. $E M B O J$ 31: $616-629$

Fan B, Malato Y, Calvisi DF, Naqvi S, Razumilava N, Ribback S, Gores G], Dombrowski F, Evert M, Chen X, Willenbring H (2012) Cholangiocarcinomas can originate from hepatocytes in mice. J Clin Invest 122: 2911-2915

He G, Dhar D, Nakagawa H, Font-Burgada J, Ogata H, Jiang Y, Shalapour S, Seki E, Yost SE, Jepsen K, Frazer KA, Harismendy O, Hatziapostolou M, Iliopoulos D, Suetsugu A, Hoffman RM, Tateishi R, Koike K, Karin M (2013) Identification of liver cancer progenitors whose malignant progression depends on autocrine IL-6 signaling. Cell 155: 384-396

Jorgensen S, Schotta G, Sorensen CS (2013) Histone $\mathrm{H} 4$ lysine 20 methylation: key player in epigenetic regulation of genomic integrity. Nucleic Acids Res 41: 2797-2806

Kreso A, Dick JE (2014) Evolution of the cancer stem cell model. Cell Stem Cell 14: 275-291

Nikolaou KC, Moulos P, Chalepakis G, Hatzis P, Oda H, Reinberg D, Talianidis I (2014) Spontaneous development of hepatocellular carcinoma with cancer stem cell properties in PR-SET7-deficient livers. EMBO J 34: $430-447$

Oishi N, Yamashita T, Kaneko S (2014) Molecular biology of liver cancer stem cells. Liver Cancer 3: $71-84$

Yamashita T, Wang XW (2013) Cancer stem cells in the development of liver cancer. J Clin Invest 123: 1911-1918

Yanger K, Zong Y, Maggs LR, Shapira SN, Maddipati R, Aiello NM, Thung SN, Wells RG, Greenbaum LE, Stanger BZ (2013) Robust cellular reprogramming occurs spontaneously during liver regeneration. Genes Deu 27: 719-724

Yimlamai D, Christodoulou C, Galli GG, Yanger K, Pepe-Mooney B, Gurung B, Shrestha K, Cahan P, Stanger BZ, Camargo FD (2014) Hippo pathway activity influences liver cell fate. Cell 157: $1324-1338$ 